\title{
Developing and validating a novel nomogram used a competing-risks model for predicting the prognosis of primary fallopian tube carcinoma: a retrospective study based on the SEER database
}

\author{
Chengzhuo $\mathrm{Li}^{1,2 \#}$, Junyuan $\mathrm{Li}^{3 \#}$, Qiao Huang ${ }^{4}$, Xiaojie Feng ${ }^{1,2}$, Fanfan Zhao ${ }^{1,2}$, Fengshuo Xu ${ }^{1,2}$, Didi Han ${ }^{1,2}$, \\ Jun Lyu ${ }^{1,2}$
}

${ }^{1}$ Department of Clinical Research, The First Affiliated Hospital of Jinan University, Guangzhou, China; ${ }^{2}$ School of Public Health, Xi'an Jiaotong University Health Science Center, Xi'an, China; ${ }^{3}$ Medical Centre of Stomatology, The First Affiliated Hospital of Jinan University, Guangzhou, China; ${ }^{4}$ Center for Evidence-Based and Translational Medicine, Zhongnan Hospital of Wuhan University, Wuhan, China

Contributions: (I) Conception and design: C Li, J Lyu; (II) Administrative support: J Lyu; (III) Provision of study materials or patients: J Li, F Xu; (IV) Collection and assembly of data: J Li, X Feng, F Zhao; (V) Data analysis and interpretation: C Li, Q Huang, D Han; (VI) Manuscript writing: All authors; (VII) Final approval of manuscript: All authors.

\#These authors contributed equally to this work.

Correspondence to: Jun Lyu. Department of Clinical Research, The First Affiliated Hospital of Jinan University, 613 West Huangpu Avenue, Tianhe District, Guangzhou 510630, China. Email: lyujun2020@jnu.edu.cn.

Background: The current prognostic methods for primary fallopian tube carcinoma (PFTC) are inadequate. This study is the first to use a competing-risks model to perform an accurate analysis of the prognostic factors for PFTC cause-specific death (CSD). We used the model to established a nomogram for the 3-, 5-, and 8-year CSD rates based on the identified prognostic factors.

Methods: This study selected 1,924 patients from the SEER (Surveillance, Epidemiology, and End Results) database. The cumulative incidence function (CIF) was used in univariate analyses, and Gray's test was used to determine the intergroup difference in the CIF. We then used the subdistribution proportional hazards model in a multivariate analysis. We finally used the prognostic factors identified in the analysis of the competing-risks model to construct a 3-, 5-, and 8-year CSD nomogram for PFTC patients. The concordance index (C-index) and calibration plots were used to evaluate the discrimination ability and consistency of the model.

Results: The subdistribution proportional hazards model showed that age, histological type, FIGO stage, and the $\log$ of the ratio between the numbers of positive and negative lymph nodes (LODDS) were independent prognostic factors for CSD. The 3-, 5-, and 8-year C-indexes were 0.744, 0.744, and 0.733 in the training cohort, and $0.737,0.748$, and 0.721 in the validation cohort. In the calibration plots, the forecast lines were very close to the reference lines.

Conclusions: This study is the first to analyze the prognostic factors for PFTC based on a competingrisks model. This model indicates that age, histological type, FIGO stage, and LODDS are significant prognostic factors affecting CSD in PFTC patients. We have also constructed the first 3-, 5-, and 8-year CSD nomogram for PFTC patients. This nomogram exhibits good discrimination ability and accuracy and can help clinicians to provide individualized prognostic analysis for PFTC patients.

Keywords: Primary fallopian tube carcinoma (PFTC); SEER; competing risk model; nomogram; cancer-specific death

Submitted Jul 19, 2020. Accepted for publication Dec 16, 2020.

doi: 10.21037/atm-20-5398

View this article at: http://dx.doi.org/10.21037/atm-20-5398 


\section{Introduction}

Primary fallopian tube carcinoma (PFTC) is a rare gynecological tumor that accounts for $0.14-1.8 \%$ of genital malignancies (1). The incidence of fallopian tube carcinoma is increasing, with the rate in North America reportedly increasing from 0.22 per 1 million females in 1999-2001 to 0.62 per 1 million females in 2011-2012 (2), and another study finding that the diagnosis rate increased fourfold from 2001 to 2014 (3). Additionally, recent studies have shown that many tumors classified as high-grade serous carcinomas of the ovary or peritoneum might originate in the fallopian tube $(4,5)$. This situation means that the incidence of PFTC might be underestimated, and hence that more attention should be paid to PFTC.

The difficulty of diagnosing PFTC early often results in a poor prognosis. A retrospective analysis found that the 5-, 8-, and 15 -year overall survival rates were $44.7 \%$, $23.8 \%$, and $18.8 \%$, respectively, while the disease-free survival rates were $27.3 \%, 17 \%$, and $14 \%$ (6). Diagnosing PFTC preoperatively is extremely difficult, whereas surgical findings enable doctors to determine a precise histological diagnosis, staging, and prognosis (1). and so all of the subjects selected for inclusion in our study had undergone surgery.

Competing risks are common in the medical arena, and the standard Cox model leads to incorrect and biased results because it does not account for competing events (7). Competing-risks models divide the outcome into three categories: censored events, the specific event, and competing-risks events (8). Competitive events are events that may affect the probability of an observed event or hinder its occurrence. When researchers pay attention to the death of specific cancer, patients may die from other cancers, suicide, traffic accidents, etc. The traditional Cox regression classifies these patients as censored data, which incorrectly estimates the cumulative incidence and HR value of the target outcome, and then deriving biased prognostic factors (7). Competitive risk becomes particularly important when analyzing the elderly population or longterm prognosis (9). The purpose of the present study was to utilize the large sample available in the Surveillance, Epidemiology, and End Results (SEER) database to identify the long-term prognostic factors for PFTC. It means that there may be a lot of competition events in the research. If Cox regression is directly used for analysis, bias will be generated. Therefore, we adopted a competitive risk model suitable for this type of data and research objectives.
Research shows that the Cox model is not suitable for identifying the risk factors in the presence of competing risks. Fine and Gray (10) proposed a subdistribution proportional hazards model, which has been widely applied (11-13). The subdistribution proportional hazards model can directly perform regression modeling on competitive risk data and then obtain more accurate prognostic factors related to specific outcomes (14). For clinical decisionmaking in the real world where competing risks do exist, actual rather than virtual risks are usually more important. The cumulative incidence provides an estimate of the percentage of patients who actually maintain the event, and the subdistribution proportional hazards model provides actual risk factors. Therefore, the competitive risk model is more meaningful for individual patient prognosis consultation and clinical resource utilization $(15,16)$.

Nomograms are widely used as prognostic devices in oncology and medicine since they allow individualized predictions and both doctors and patients find their visualizations easy to understand (17). We are not aware of any previous research that has constructed a PFTC prognostic nomogram, and so another purpose of this research was using the prognostic factors derived from the competing-risks model to construct and validate the 3-, 5-, and 8-year cause-specific death (CSD) nomogram for PFTC patients, which can be used to guide clinical decisions. We present the following article in accordance with the TRIPOD reporting checklist (available at http:// dx.doi.org/10.21037/atm-20-5398).

\section{Methods}

The study was conducted in accordance with the Declaration of Helsinki (as revised in 2013). Because the information in the SEER database does not require the patient's explicit consent, the study is waived from ethical approval. The informed patient consent is not required due to the retrospective nature of the study.

\section{Patient selection}

This study analyzed data obtained from the SEER database. The SEER Program collects and publishes cancer incidence and survival data from population-based cancer registries covering approximately $34 \%$ of the US population. This large database provides relatively accurate clinical information (18). We search the database from SEER 18 Regs Custom Data (with additional treatment fields), Nov 


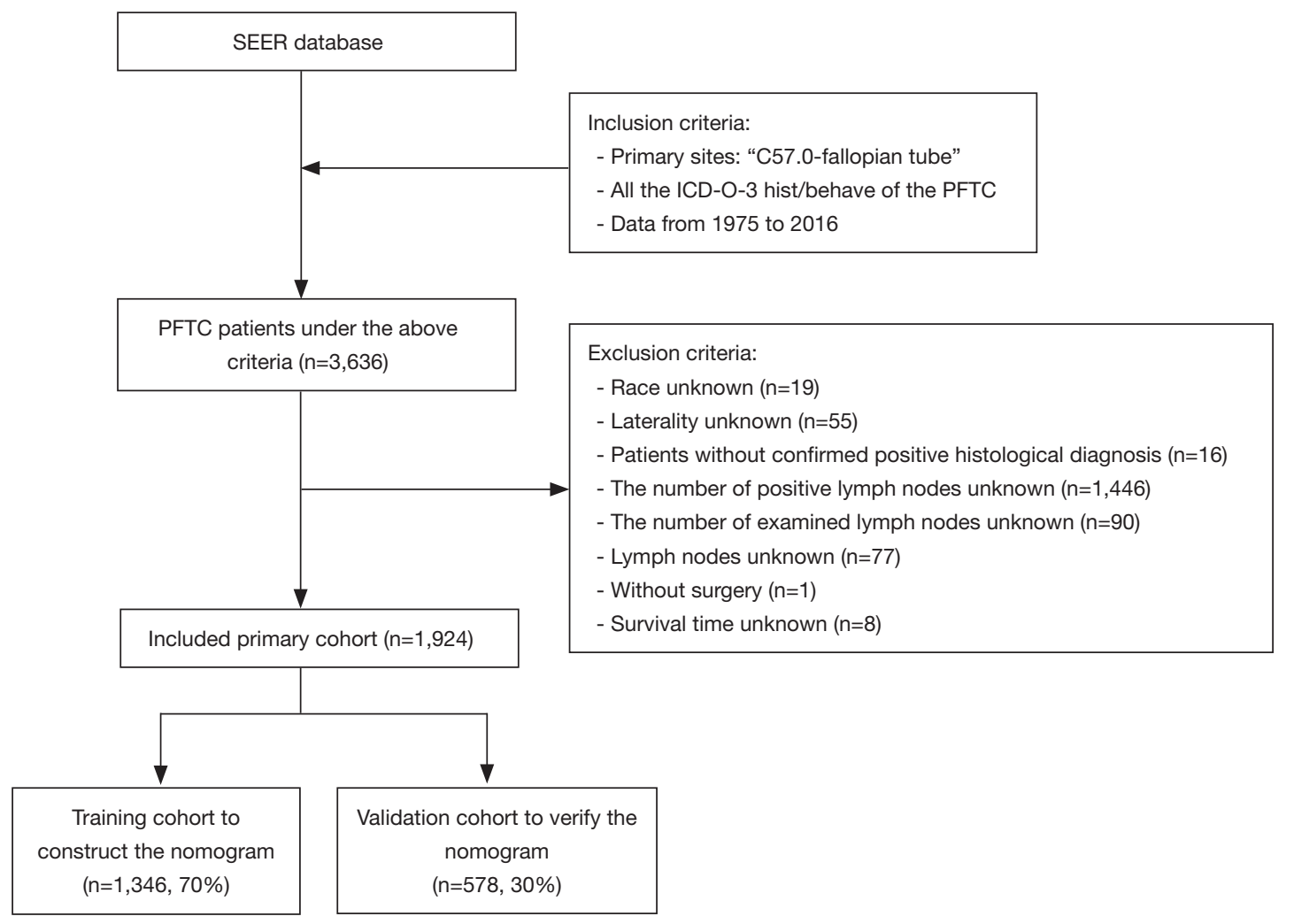

Figure 1 Data selection flowchart.

2018 Sub (1975-2016 varying), including for chemotherapy data. All of the data were downloaded using SEER software (version 8.3.6). Because some of the SEER research data is publicly available, no informed consent or institutional review board approval was required.

\section{Inclusion and exclusion criteria}

We extracted PFTC patients from the SEER database using the ICD-O-3 "C57.0-Fallopian tube" code, with all of the ICD-O-3 histology and behavior codes related to PFTC included. The age at diagnosis, year of diagnosis, race, sex, and marital status were selected as demographic characteristics. All subjects selected in our study had undergone surgery. The following pathological features were also included: laterality, tumor size, FIGO stage, treatment (radiotherapy status and chemotherapy status), lymph nodes ratio (LNR), and the log of the ratio between the numbers of positive and negative lymph nodes (LODDS). LNR is calculated by dividing the number of positive lymph nodes (pnod) by the total number of examined lymph nodes (tnod), while LODDS is calculated as $\log (\operatorname{pnod}+0.5) /(\operatorname{tnod}-\operatorname{pnod}+0.5)$, where 0.5 is added to the denominator to avoid an infinite number and thus also to the numerator to reduce bias (19).

The age at diagnosis and the year of diagnosis were analyzed as continuous variables. The tumor size was divided into the following four categories based on the maximum diameter: $<2,2-4,>4 \mathrm{~cm}$, and unknown. We divided PFTC into five categories of histological types: 1 , serous cystadenocarcinoma; 2, papillary serous cystadenocarcinoma; 3 , endometrioid carcinoma; 4, adenocarcinoma; and 5 , others. Since data from the FIGO staging system are not directly entered into the SEER database, we integrated the FIGO staging system for fallopian tube carcinoma using the equivalent TNM classifications (20).

We excluded some data to ensure the accuracy of the study, such as patients without a confirmed positive histological diagnosis, as well as those with unknown indicators. We eventually retained 1,924 patients for inclusion in further analyses. The data screening and sorting processes are shown in detail in Figure 1. The study outcomes included survival, CSD, and death due to other 
causes (DOC). The survival time was measured in months.

\section{Statistical analysis}

We randomly divided the 1,924 screened patients with fallopian tube carcinoma into a training group $(70 \%$, $\mathrm{n}=1,346)$ and a validation group $(30 \%, \mathrm{n}=578)$ using $\mathrm{R}$ software. We used SPSS software to describe the general situation of the two patient cohorts. Continuous data are presented as quartiles, and categorical data are presented as frequencies and percentages. We used the chi-square test and the t-test to test the homogeneity between the two groups of data. Since there is no universal standard for classifying LNR and LODDS, we used X-tile (Yale University School of Medicine, New Haven, CT, USA) to determine the optimal cutoffs for dividing LNR and LODDS into three levels based on the minimum $P$ values in the log-rank test and the highest specificity and sensitivity $(21,22)$. The optimal cutoffs were used to classify LNR into the following three groups: LNR I (0-0.02), LNR II (0.03-0.50), and LNR III (0.51-1). Similarly, LODDS was classified into the following three groups: LODDS I ( -2.13 to -1.07$)$, LODDS II (-1.06 to -0.49$)$, and LODDS III $(-0.48$ to 1.77$)$.

In the competing-risks analysis, DOC was treated as an event competing with CSD. The cumulative incidence function (CIF) was used for univariate analyses, and Gray's test was used to determine the intergroup difference in the CIF. We used the CIF to calculate the CSD and DOC probabilities among PFTC patients. For the multivariate analysis, we used the subdistribution proportional hazards model to determine the prognostic factors for PFTC. The traditional Cox regression analysis model was used for comparative analysis.

Finally, we used the prognostic factors identified in the analysis of the competing-risks model to construct a 3-, 5-, and 8-year CSD nomogram for PFTC patients with $\mathrm{R}$ software. After constructing the nomogram, we used the concordance index (C-index) and calibration plots to evaluate the discrimination ability and consistency of the model.

All statistical tests were conducted using SPSS (version 23.0, IBM Corporation, Armonk, NY, USA), R software (version 3.5.3, The R Foundation for Statistical Computing, Vienna, Austria; http://www.r-project.org), and SAS (version 9.4, SAS Institute, Cary, NC, USA). Probability values of $\mathrm{P}<0.05$ in two-sided tests were considered statistically significant.

\section{Results}

\section{Basic characteristics}

The basic demographic and pathological characteristics of the 1,924 patients are listed in Table 1 . The median age at diagnosis was 62.0 years (interquartile range $=54.0-70.0$ years) in the training cohort and 62.5 years (interquartile range $=55.0-69.3$ years) in the validation cohort. Most patients in the training and validation cohorts were white $(85.7 \%$ and $85.5 \%$, respectively) and married (81.9\% and $81.5 \%$, respectively). Regarding pathological features, most of the tumors were at histological grade III (about $45 \%$ ), unilateral (about 90\%), and larger than $4 \mathrm{~cm}$ (about 30\%) in both cohorts. The most common histological type was serous cystadenocarcinoma (about 50\%), followed by papillary serous cystadenocarcinoma (about 20\%). Approximately $40 \%$ of patients were at FIGO stage III. Most of the patients in both cohorts had received adjuvant chemotherapy, while only a few had received adjuvant radiotherapy. LNR was $\leq 0.02$ and LODDS was $\leq-1.07$ in most patients (about $70 \%$ and $55 \%$, respectively). None of the study variables differed significantly between the training and validation cohorts, demonstrating that the two cohorts of data were balanced and comparable.

\section{Univariate analyses}

Univariate analyses were applied to the study variables to calculate the 3-, 5-, and 8-year CIF values of CSD and DOC in the training cohort $(n=1,346)$. In the results of univariate analyses, variables with a $\mathrm{P}$ value less than 0.1 are considered meaningful. The results showed that the age at diagnosis, year of diagnosis, marital status, laterality, histological type, FIGO stage, adjuvant chemotherapy, LNR, and LODDS were significantly related to CSD. Meanwhile, age and tumor size were significantly related to DOC, while race, histological grade, and adjuvant radiotherapy were not related to either outcome. The CIF and $\mathrm{P}$ values are presented in Table 2. The CIF curves for significant variables are shown in Figure 2.

\section{Multivariate analysis}

The meaningful variables identified in the univariate analyses were included in the multivariate analysis, and the subdistribution proportional hazards model and the Cox regression model were constructed. The analysis results are 
Table 1 The basic characteristics of the patients

\begin{tabular}{|c|c|c|c|}
\hline Variable & Training cohort & Validation cohort & $P$ \\
\hline Age mean (range) & $62.0(54.0-70.0)$ & $62.5(55.0-69.3)$ & 0.901 \\
\hline Year of diagnosis mean (range) & 2011 (2008-2013) & 2011 (2008-2013) & 0.699 \\
\hline Race, n (\%) & & & 0.174 \\
\hline Black & $87(6.5)$ & $28(4.8)$ & \\
\hline Other & $105(7.8)$ & $56(9.7)$ & \\
\hline Marital status, n (\%) & & & 0.823 \\
\hline Married & $1,102(81.9)$ & $471(81.5)$ & \\
\hline 1 & $38(2.8)$ & $23(4.0)$ & \\
\hline II & $115(8.5)$ & $54(9.3)$ & \\
\hline III & $596(44.3)$ & $263(45.5)$ & \\
\hline IV & $427(31.7)$ & $168(29.1)$ & \\
\hline Others & $170(12.6)$ & $70(12.1)$ & \\
\hline Laterality, n (\%) & & & 0.424 \\
\hline One & $1,244(92.4)$ & $528(91.3)$ & \\
\hline Unknown & $348(25.9)$ & $164(28.4)$ & \\
\hline Histological type, n (\%) & & & 0.602 \\
\hline Serous cystadenocarcinoma & $717(53.3)$ & $297(51.4)$ & \\
\hline Papillary serous cystadenocarcinoma & $255(18.9)$ & $112(19.4)$ & \\
\hline Endometrioid carcinoma & $109(8.1)$ & $44(7.6)$ & \\
\hline Adenocarcinoma & $81(6.0)$ & $46(8.0)$ & \\
\hline Others & $184(13.7)$ & $79(13.7)$ & \\
\hline FIGO stage, n (\%) & & & 0.775 \\
\hline I & $376(27.9)$ & $168(29.1)$ & \\
\hline II & $250(18.6)$ & 96 (16.6) & \\
\hline III & $561(41.7)$ & $244(42.2)$ & \\
\hline IV & $159(11.8)$ & $70(12.1)$ & \\
\hline
\end{tabular}

Table 1 (continued) 
Table 1 (continued)

\begin{tabular}{lcc}
\hline Variable & Training cohort & Validation cohort \\
\hline Adjuvant radiotherapy, $\mathrm{n}(\%)$ & $26(1.9)$ & $12(2.1)$ \\
Yes & $1,320(98.1)$ & $566(97.9)$ \\
NO/Unknown & & \\
Adjuvant chemotherapy, $\mathrm{n}(\%)$ & $1,045(77.6)$ & $428(74.0)$ \\
Yes & $301(22.4)$ & $150(26.0)$ \\
NO/Unknown & & \\
LNR $n(\%)$ & $912(67.8)$ & $402(69.6)$ \\
{$[0,0.02]$} & $282(21.0)$ & $106(18.3)$ \\
{$[0.03,0.52]$} & $152(11.3)$ & $70(12.1)$ \\
{$[0.53,1]$} & & \\
LODDS $n(\%)$ & $737(54.8)$ & $317(54.8)$ \\
{$[-2.13,-1.07]$} & $274(20.4)$ & $116(20.1)$ \\
{$[-1.06,-0.49]$} & $335(24.9)$ & $145(25.1)$ \\
{$[-0.48,1.77]$} & 0.408 \\
\hline
\end{tabular}

LNR, lymph nodes ratio; LODDS, log of odds between the number of positive lymph nodes and the number of negative lymph nodes.

Table 2 The cumulative incidences of CSD and DOC

\begin{tabular}{|c|c|c|c|c|c|c|c|c|c|c|}
\hline Variables & \multicolumn{3}{|c|}{ Cause-specific death (\%) } & - Gray's test & $\mathrm{P}$ & \multicolumn{3}{|c|}{ Death due to other causes (\%) } & Gray's test & $\mathrm{P}$ \\
\hline Age & - & - & - & 106.378 & $<0.001$ & - & - & - & 285.616 & $<0.001$ \\
\hline Year of diagnosis & - & - & - & 22.927 & 0.018 & - & - & - & 14.641 & 0.200 \\
\hline Race & & & & 0.335 & 0.846 & & & & 0.609 & 0.738 \\
\hline Black & 10.348 & 20.578 & 28.265 & & & 10.872 & 12.594 & 20.886 & & \\
\hline Other & 13.822 & 24.218 & 29.511 & & & 5.361 & 8.977 & 14.179 & & \\
\hline Marital status & & & & 7.099 & 0.029 & & & & 2.171 & 0.338 \\
\hline Married & 14.467 & 23.205 & 28.122 & & & 7.025 & 10.435 & 15.024 & & \\
\hline Grade & & & & 8.111 & 0.088 & & & & 4.951 & 0.292 \\
\hline I & 6.231 & 6.231 & 6.231 & & & 5.569 & 14.091 & 14.091 & & \\
\hline II & 10.424 & 18.849 & 23.333 & & & 5.669 & 9.306 & 12.921 & & \\
\hline III & 14.003 & 22.053 & 28.292 & & & 6.214 & 8.898 & 14.924 & & \\
\hline IV & 14.390 & 26.025 & 29.470 & & & 6.334 & 9.453 & 16.238 & & \\
\hline Others & 14.646 & 19.021 & 23.134 & & & 14.071 & 16.449 & 18.279 & & \\
\hline
\end{tabular}

Table 2 (continued) 
Table 2 (continued)

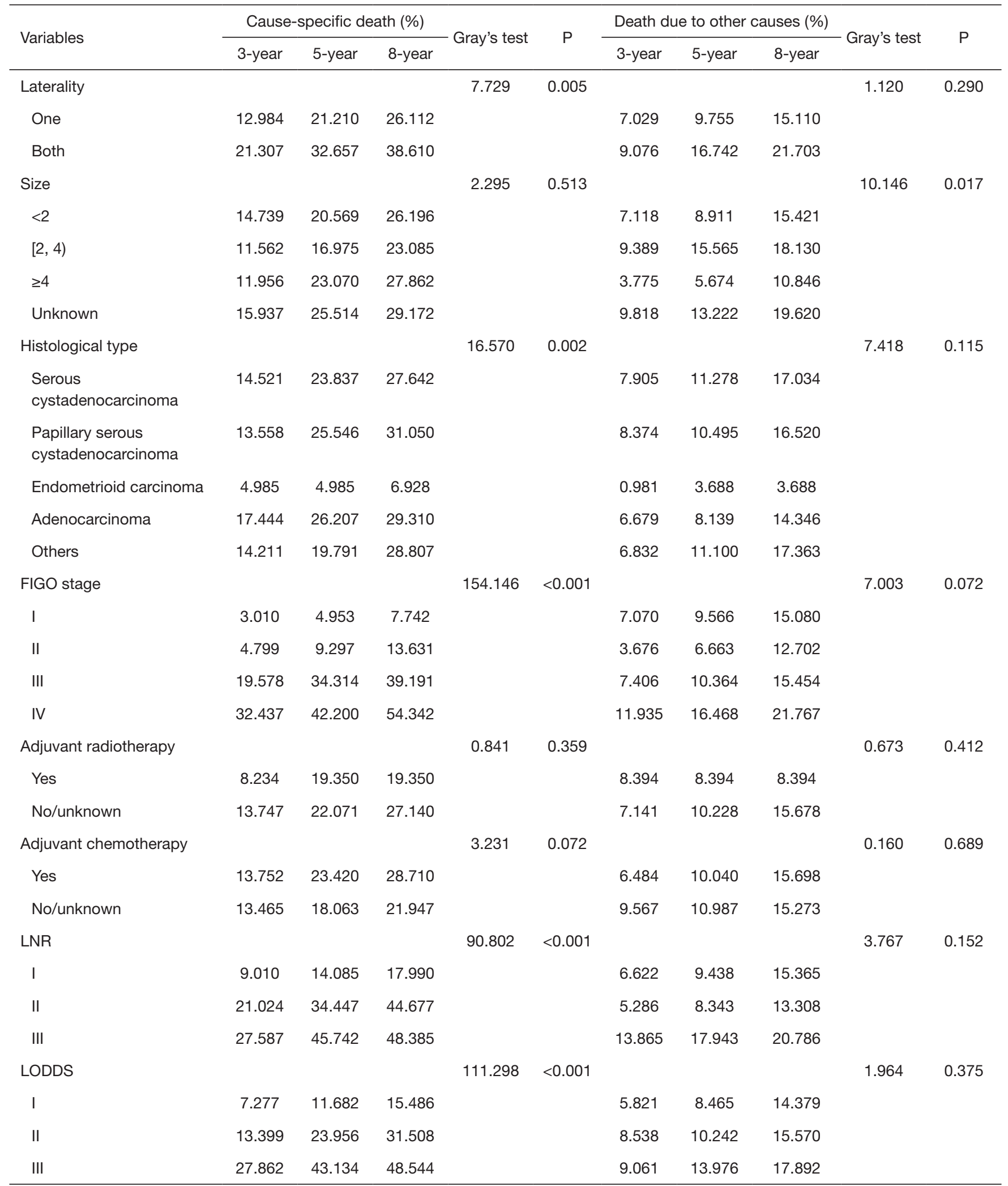

LNR, lymph nodes ratio; LODDS, log of odds between the number of positive lymph nodes and the number of negative lymph nodes. 


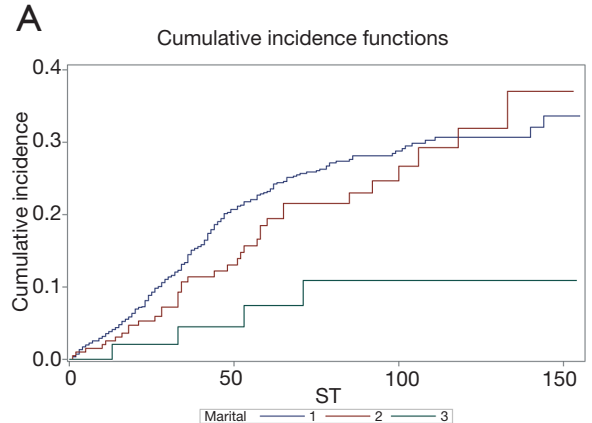

D

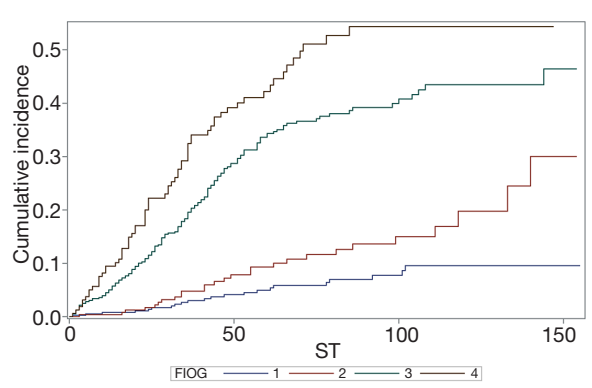

G

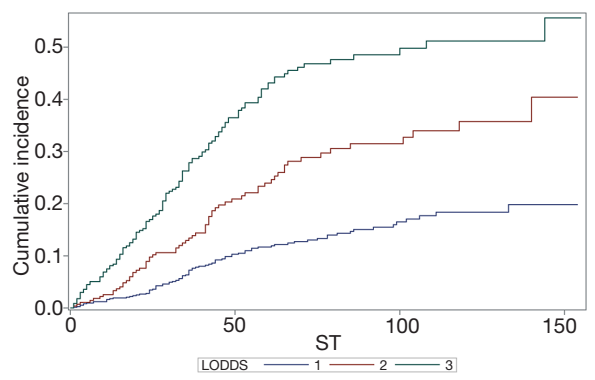

B

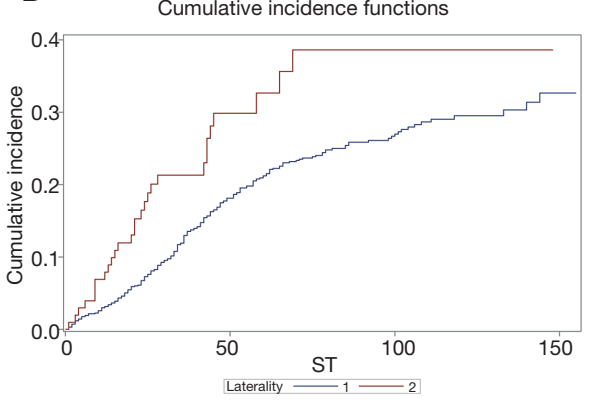

E
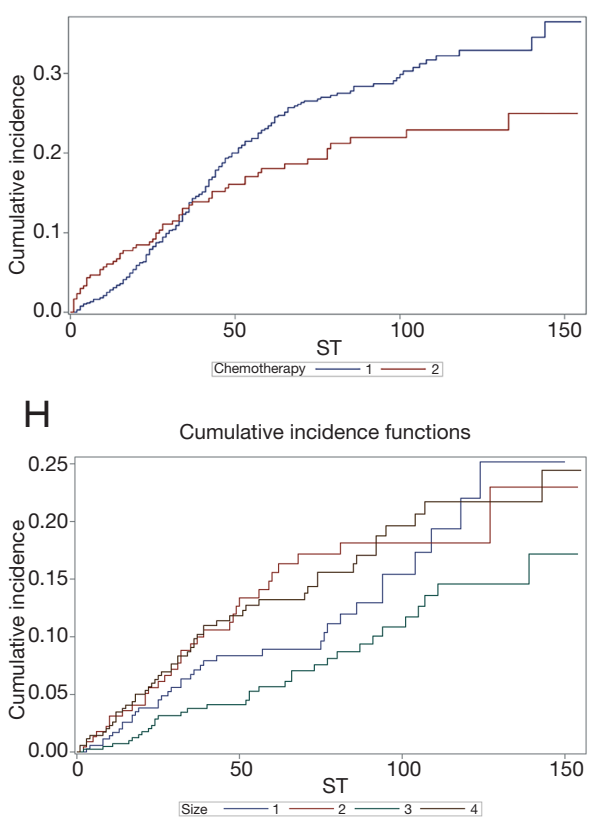

C

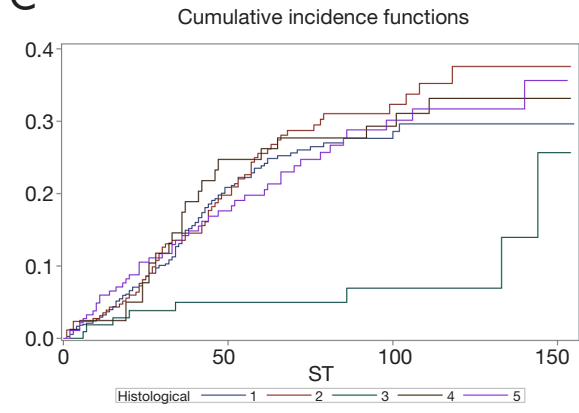

$\mathrm{F}$

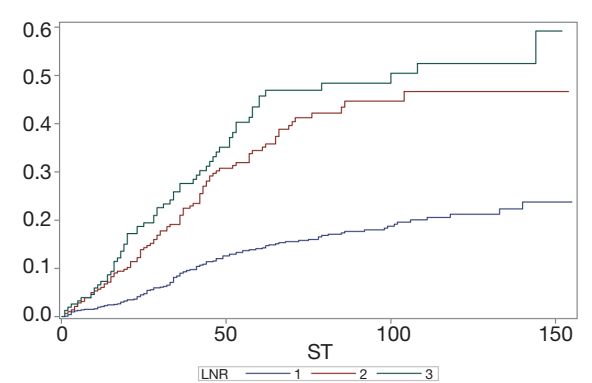

Figure 2 The CIF curves for significant variables of cause-specific death (A,B,C,D,E,F,G) and death due to other causes (H).

presented in detail in Table 3.

The multivariate competing-risks analysis indicated that the significant prognostic factors affecting PFTC were the age at diagnosis (HR $=1.016,95 \%$ CI: 1.005-1.027), histological type (relative to Serous cystadenocarcinoma: $\mathrm{HR}=1.613$ for adenocarcinoma, 95\% CI: 1.022-2.545), FIGO stage (relative to stage I: $\mathrm{HR}=1.881$ for stage II, $95 \%$ CI: $1.067-3.316$; HR $=4.504$ for stage III, $95 \%$ CI: $2.735-$ 7.418; HR $=6.324$ for stage IV, 95\% CI: 3.723-10.740), and LODDS (relative to LODDS I: HR $=2.161$ for LODDS III, 95\% CI: 1.411-3.312). The Cox regression model showed that the age at diagnosis, year of diagnosis, histological type, FIGO stage, and LODDS were prognostic factors for
PFTC $(\mathrm{P}<0.05)$.

\section{Constructing and verifying the nomogram}

We used the above results from the multivariate analysis of CSD to construct the 3-, 5-, and 8-year nomogram for PFTC patients shown in Figure 3. The figure shows that the probability of CSD was most affected by the FIGO stage, followed by the age at diagnosis, histological type, and LODDS. Total points are obtained by adding the scores corresponding to the patient's prognostic factors, which clinicians can use to predict 3-, 5-, and 8-year survival rates for individual patients.

The nomogram constructed using the training cohort $(n=1,346)$ was verified using the validation cohort $(n=578)$. 
Table 3 Meaningful variables by subdistribution proportional hazards model and Cox regression model

\begin{tabular}{|c|c|c|c|c|c|c|c|c|}
\hline Variables & \multicolumn{4}{|c|}{ Subdistribution proportional hazards model } & \multicolumn{4}{|c|}{ Cox regression model } \\
\hline Age & 0.016 & 1.016 & $1.005-1.027$ & 0.005 & 0.034 & 1.035 & $1.025-1.044$ & $<0.001$ \\
\hline Year of diagnosis & 0.014 & 1.014 & $0.975-1.055$ & 0.476 & 0.042 & 1.043 & $1.005-1.081$ & 0.025 \\
\hline \multicolumn{9}{|l|}{ Marital status } \\
\hline Others & -0.915 & 0.401 & $0.155-1.035$ & 0.059 & -0.147 & 0.863 & $0.492-1.515$ & 0.608 \\
\hline \multicolumn{9}{|l|}{ Laterality } \\
\hline One & \multicolumn{4}{|c|}{ Reference } & \multicolumn{4}{|c|}{ Reference } \\
\hline$[2,4)$ & -0.169 & 0.845 & $0.564-1.265$ & 0.413 & 0.069 & 1.071 & $0.790-1.453$ & 0.657 \\
\hline$\geq 4$ & 0.052 & 1.054 & $0.752-1.477$ & 0.762 & -0.045 & 0.956 & $0.730-1.252$ & 0.742 \\
\hline Unknown & -0.024 & 0.976 & $0.700-1.362$ & 0.887 & 0.157 & 1.170 & $0.902-1.518$ & 0.237 \\
\hline \multicolumn{9}{|l|}{ Histological type } \\
\hline Serous cystadenocarcinoma & \multicolumn{4}{|c|}{ Reference } & \multicolumn{4}{|c|}{ Reference } \\
\hline $\begin{array}{l}\text { Papillary serous } \\
\text { cystadenocarcinoma }\end{array}$ & 0.173 & 1.189 & $0.877-1.612$ & 0.266 & 0.183 & 1.201 & $0.940-1.535$ & 0.143 \\
\hline Endometrioid carcinoma & -0.517 & 0.596 & $0.290-1.225$ & 0.159 & -0.679 & 0.507 & $0.291-0.885$ & 0.017 \\
\hline IV & 1.844 & 6.324 & $3.723-10.740$ & $<0.001$ & 1.148 & 3.151 & $2.189-4.536$ & $<0.001$ \\
\hline
\end{tabular}

Adjuvant chemotherapy

Yes

Reference

No/unknown

LNR

I

II

III

LODDS

I

II

III
0.142

1.152

0.830-1.599

0.398

Reference

$-0.082$

$-0.134$

0.921

0.640-1.327

0.874

0.546-1.399

0.660

0.575

Reference

\begin{abstract}
0.359
\end{abstract}
$1.432 \quad 0.996-2.059 \quad 0.053$

0.771

$2.161 \quad 1.411-3.312<0.001$
0.146

Reference

$1.157 \quad 0.908-1.474 \quad 0.237$

Reference

$-0.094$

0.098

$0.910 \quad 0.678-1.222$

0.531

1.102

$0.757-1.606$

0.611

HR, hazard ratio; LNR, lymph nodes ratio; LODDS, log of odds between the number of positive lymph nodes and the number of negative lymph nodes. 


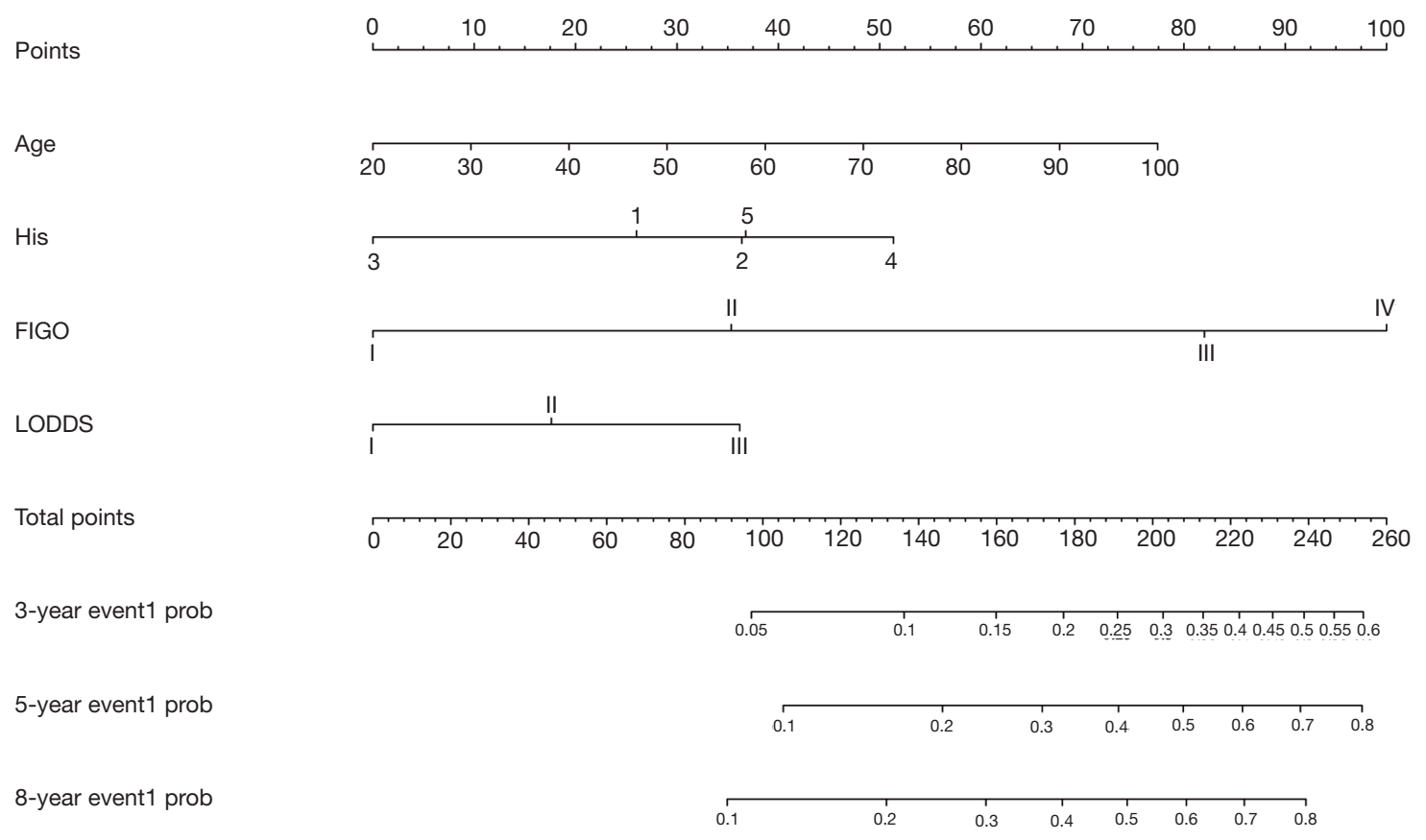

Figure 3 Nomogram based on the competing risk analysis to predict cancer-specific death probabilities at 3, 5, and 8 years for PFTC patients. PFTC, primary fallopian tube carcinoma.

The 3-, 5-, and 8-year C-indexes were 0.744, 0.744, and 0.733 , respectively, in the training cohort, and $0.737,0.748$, and 0.721 in the validation cohort. The good discrimination ability of the model is demonstrated by all of these values exceeding 0.7 . We also used calibration plots to test the prediction accuracy of the model. As shown in Figure 4, the 3-, 5-, and 8-year predicted values used in the training and verification cohorts were very close to the actual values, indicating that the model is very accurate.

\section{Discussion}

While PFTC is relatively rare in female reproductive organs, recent studies have shown that the incidence of fallopian tube carcinoma may be underestimated $(23,24)$. Previous research on PFTC has been insufficient, which prompted us to analyze the prognostic factors for PFTC using a large amount of data available in the SEER database, and construct a nomogram for improving the prediction capabilities for individual patients.

In our study, competition events accounted for about $35 \%$ of the total deaths, accounting for a very high proportion, which indicates that competition events are likely to occur when analyzing the PFTC long- term prognosis. Most previous studies have used the traditional Cox proportional hazards model to analyze the PFTC prognostic factors (25-28). These studies did not take competitive risk into account when considering the survival rate as the outcome. Therefore, these studies may overestimate the cumulative incidence rate and cause some factors to be erroneously classified as prognostic factors $(16,29)$.

The multivariate analysis of the competing-risks model performed in the present study revealed that the age at diagnosis, histological type, FIGO stage, and LODDS were significant prognostic factors affecting PFTC. In contrast to the competing-risks model, the Cox regression model showed that the year of diagnosis is an additional prognostic factor for PFTC. This discrepancy between the two models indicates that the Cox regression model overestimates the HR value of most variables, and even makes the year of diagnosis factor a false-positive factor, and becoming a statistically significant prognostic factor. It is unwise to consume medical supplies or conduct research on such false-positive factors. The apparent effect of the year of diagnosis may be simply due to the natural progression of the disease (30). causing the illusion that this factor is related to CSD. This phenomenon may also exist for other 
A

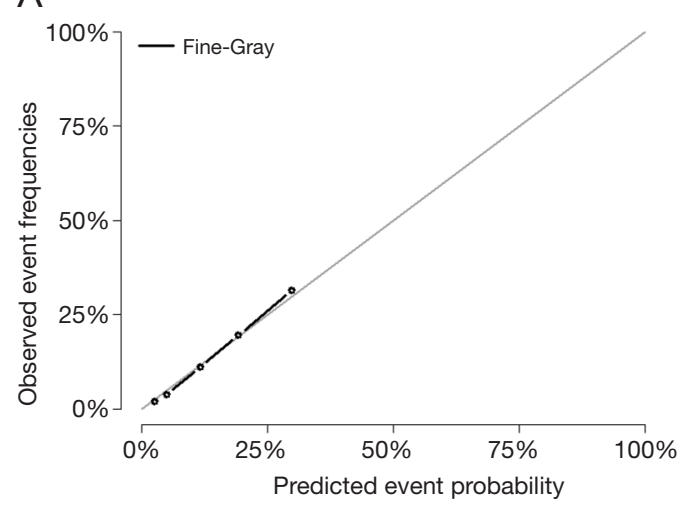

B

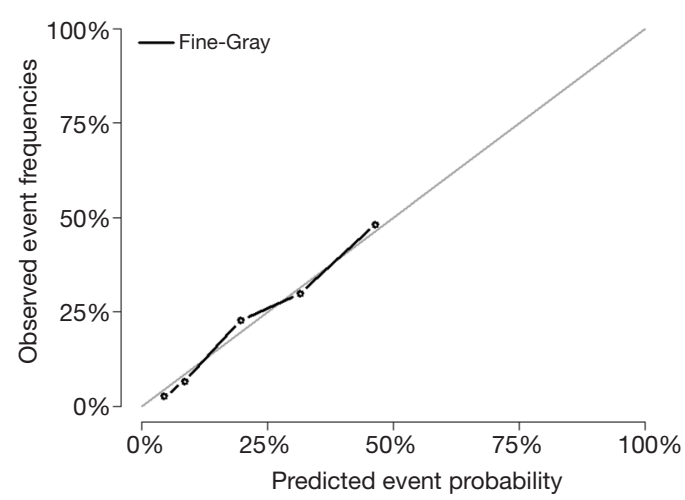

C

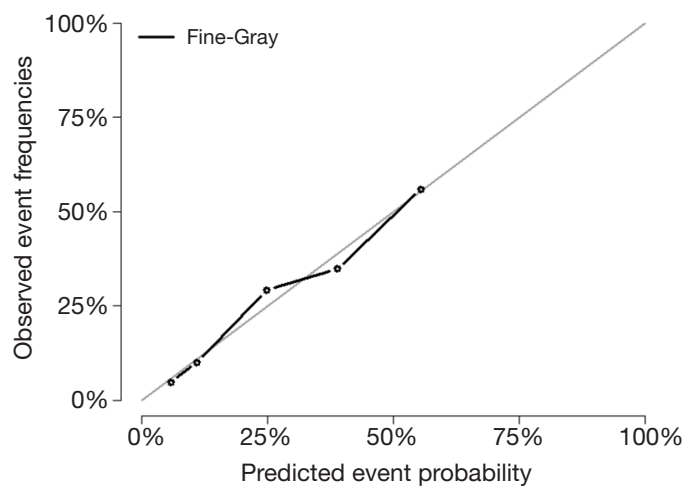

D

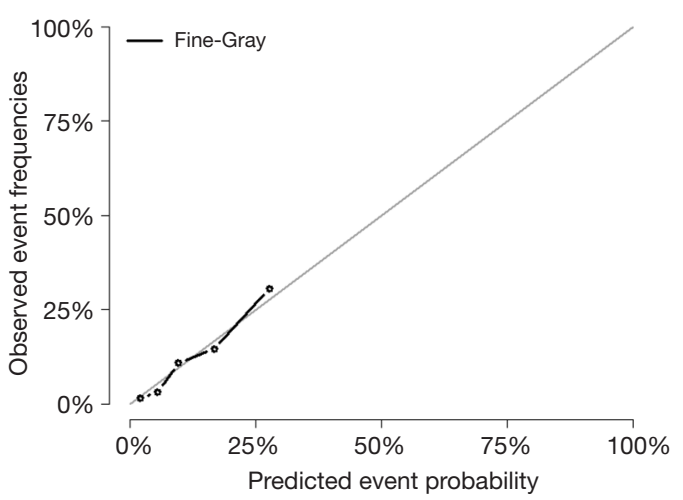

E

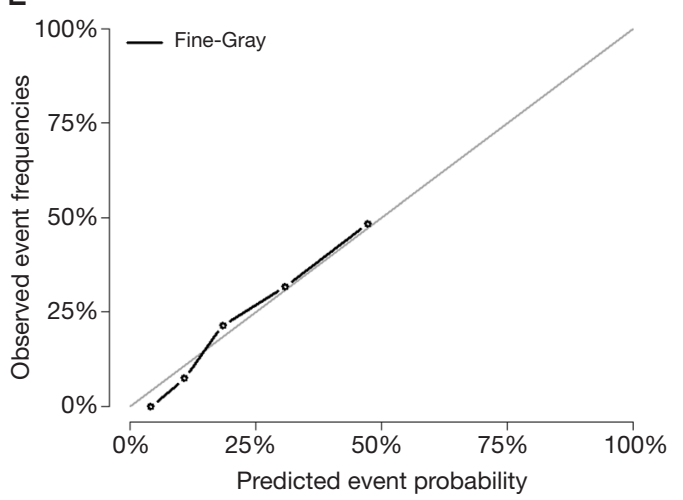

$\mathrm{F}$

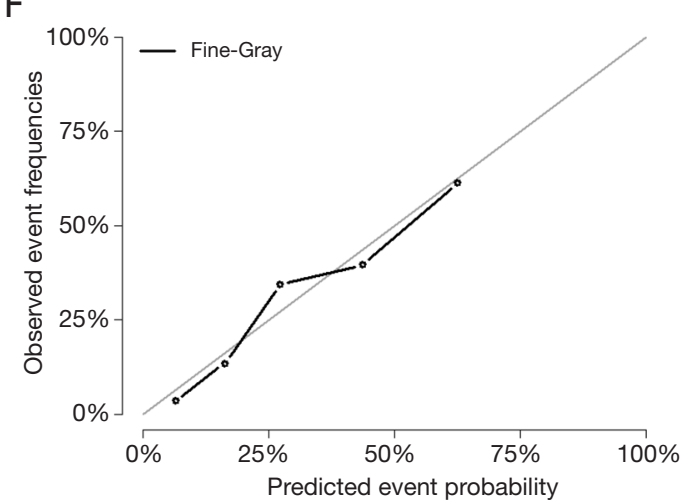

Figure 4 Calibration curves. Calibration curves for 3-, 5-, and 8-year calibration plots of the training (A,B,C) and validation (D,E,F) cohort.

cancer prognostic factors. Analyzing a competing-risks model can exclude some factors related to DOC, which is important for ensuring that optimal clinical interventions are applied.

This study is the first to use the prognostic factors identified in a multivariate analysis to construct a nomogram that includes competing risks in PFTC patients. A 15-year overview study found that the FIGO stage was the most powerful predictor of outcome in females with PFTC (31). while other previous studies have also shown that age represents an important prognostic variable for PFTC survival (26); our results are consistent with these reports. However, it remains inconclusive about whether the histological type is a prognostic factor for PFTC, with no study has analyzed the prognosis in a large sample. We found that histological type is a prognostic factor, with the 
prognosis being best for endometrioid carcinoma and worst for adenocarcinoma. We consider that more studies are needed to determine the effect of histological type on the prognosis of PFTC patients.

LNR and LODDS are novel indicators of the lymph node status, and some recent studies have applied these parameters when analyzing the cancers prognostic factors such as rectal cancer, colon cancer, and gastric adenocarcinoma (32-34). However, no previous studies have evaluated them in PFTC patients. In our univariate analyses, both LNR and LODDS were significantly associated with CSD. However, in the multivariate analysis, LNR was no longer a prognostic factor, which indicates that LODDS is a better prognostic factor than LNR. This is consistent with conclusions based on a previous study of stage III colon cancer (35). This may be because LNR values of 0 or 1 do not fully reflect the specific condition of the lymph nodes. However, the accuracy of LODDS as a prognostic factor needs to be verified in further experimental studies.

After successfully constructing a nomogram for the prognosis of PFTC, we verified it. In the discrimination test, the $\mathrm{C}$-indexes both in the training and validation cohorts exceeded 0.7 , which indicates good discriminatory accuracy. Besides, in the consistency test, the forecast lines in the calibration plot were very close to the reference lines. These indicators demonstrate that our model is highly accurate and exhibits good discrimination ability, and can be used to guide clinical professionals in making predictions of the prognosis of individual patients $(36,37)$.

This study was subject to certain limitations. First, it had a retrospective design, which inevitably leads to selection bias. Second, we used internal data for the verification process, and so additional external data should be used to further verify the accuracy of the model. Third, some possible prognostic factors such as HER-2/neu expression and $\mathrm{p} 53$ alteration $(38,39)$ are not included in the SEER database, which will require a special experimental design in future research.

\section{Conclusions}

This study is the first to analyze the prognostic factors for PFTC based on a competing-risks model. This model revealed that the age at diagnosis, histological type, FIGO stage, and LODDS were significant prognostic factors affecting CSD in PFTC patients. This is also the first study to construct a 3-, 5-, and 8-year CSD nomogram for PFTC patients. This nomogram has good discrimination ability and accuracy and can help clinicians to apply betterindividualized treatments to PFTC patients.

\section{Acknowledgments}

Funding: The study was supported by The National Social Science Foundation of China (grant No. 16BGL183).

\section{Footnote}

Reporting Checklist: The authors have completed the TRIPOD reporting checklist. Available at http://dx.doi. org/10.21037/atm-20-5398

Conflicts of Interest: All authors have completed the ICMJE uniform disclosure form (available at http://dx.doi. org/10.21037/atm-20-5398). All authors report grants from National Social Science Foundation of China (grant No. 16BGL183), during the conduct of the study.

Ethical Statement: The authors are accountable for all aspects of the work in ensuring that questions related to the accuracy or integrity of any part of the work are appropriately investigated and resolved. The study was conducted in accordance with the Declaration of Helsinki (as revised in 2013). Because the information in the SEER database does not require the patient's explicit consent, the study is waived from ethical approval. The informed patient consent is not required due to the retrospective nature of the study.

Open Access Statement: This is an Open Access article distributed in accordance with the Creative Commons Attribution-NonCommercial-NoDerivs 4.0 International License (CC BY-NC-ND 4.0), which permits the noncommercial replication and distribution of the article with the strict proviso that no changes or edits are made and the original work is properly cited (including links to both the formal publication through the relevant DOI and the license). See: https://creativecommons.org/licenses/by-nc-nd/4.0/.

\section{References}

1. Kalampokas E, Kalampokas T, Tourountous I. Primary fallopian tube carcinoma. Eur J Obstet Gynecol Reprod Biol 2013;169:155-61.

2. Trabert B, Coburn SB, Mariani A, et al. Reported incidence and survival of fallopian tube carcinomas: 
A population-based analysis from the north american association of central cancer registries. J Natl Cancer Inst 2018;110:750-7.

3. Liao CI, Chow S, Chen LM, et al. Trends in the incidence of serous fallopian tube, ovarian, and peritoneal cancer in the us. Gynecol Oncol 2018;149:318-23.

4. Kurman RJ, Shih Ie M. Pathogenesis of ovarian cancer: Lessons from morphology and molecular biology and their clinical implications. Int J Gynecol Pathol 2008;27:151-60.

5. Pectasides D, Pectasides E, Economopoulos T. Fallopian tube carcinoma: A review. Oncologist 2006;11:902-12.

6. Wolfson AH, Tralins KS, Greven KM, et al. Adenocarcinoma of the fallopian tube: results of a multiinstitutional retrospective analysis of 72 patients. Int $\mathrm{J}$ Radiat Oncol Biol Phys 1998;40:71-6.

7. Kim HT. Cumulative incidence in competing risks data and competing risks regression analysis. Clin Cancer Res 2007;13:559-65.

8. Satagopan JM, Ben-Porat L, Berwick M, et al. A note on competing risks in survival data analysis. Br J Cancer 2004;91:1229-35.

9. Berry SD, Ngo L, Samelson EJ, et al. Competing risk of death: An important consideration in studies of older adults. J Am Geriatr Soc 2010;58:783-7.

10. Fine JP, Gray RJ. A proportional hazards model for the subdistribution of a competing risk. J Am Stat Assoc 1999;94:496-509.

11. Kattan MW, Heller G, Brennan MF. A competing-risks nomogram for sarcoma-specific death following local recurrence. Stat Med 2003;22:3515-25.

12. Zhou H, Zhang Y, Qiu Z, et al. Nomogram to predict cause-specific mortality in patients with surgically resected stage i non-small-cell lung cancer: A competing risk analysis. Clin Lung Cancer 2018;19:e195-e203.

13. Ryberg M, Nielsen D, Osterlind K, et al. Predictors of central nervous system metastasis in patients with metastatic breast cancer. A competing risk analysis of 579 patients treated with epirubicin-based chemotherapy. Breast Cancer Res Treat 2005;91:217-25.

14. Lau B, Cole SR, Gange SJ. Competing risk regression models for epidemiologic data. Am J Epidemiol 2009; 170:244-56.

15. Grunkemeier GL, Anderson RP, Miller DC, et al. Timerelated analysis of nonfatal heart valve complications: cumulative incidence (actual) versus Kaplan-Meier (actuarial). Circulation 1997;96:II-70-4; discussion II-74-5.

16. Wolbers M, Koller MT, Witteman JC, et al. Prognostic models with competing risks: Methods and application to coronary risk prediction. Epidemiology 2009;20:555-61.

17. Balachandran VP, Gonen M, Smith JJ, et al. Nomograms in oncology: More than meets the eye. Lancet Oncol 2015;16:e173-80.

18. Yang J, Li Y, Liu Q, et al. Brief introduction of medical database and data mining technology in big data era. J Evid Based Med 2020;13:57-69.

19. Sun Z, Xu Y, Li de M, et al. Log odds of positive lymph nodes: A novel prognostic indicator superior to the numberbased and the ratio-based n category for gastric cancer patients with r0 resection. Cancer 2010;116:2571-80.

20. Berek JS, Kehoe ST, Kumar L, et al. Cancer of the ovary, fallopian tube, and peritoneum. Int J Gynaecol Obstet 2018;143:59-78.

21. Camp RL D-FM, Rimm DL. X-tile: A new bio-informatics tool for biomarker assessment and outcome-based cutpoint optimization. Clin Cancer Res 2004;10:7252-9.

22. Wang W, Xu DZ, Li YF, et al. Tumor-ratio-metastasis staging system as an alternative to the 7 th edition uicc tnm system in gastric cancer after $\mathrm{d} 2$ resection--results of a single-institution study of 1343 chinese patients. Ann Oncol 2011;22:2049-56.

23. Rosenblatt KA, Weiss NS, Schwartz SM. Incidence of malignant fallopian tube tumors. Gynecol Oncol 1989;35:236-9.

24. Goodman MT, Shvetsov YB. Incidence of ovarian, peritoneal, and fallopian tube carcinomas in the united states, 1995-2004. Cancer Epidemiol Biomarkers Prev 2009;18:132-9.

25. Hefler LA, Rosen AC, Graf AH, et al. The clinical value of serum concentrations of cancer antigen 125 in patients with primary fallopian tube carcinoma: A multicenter study. Cancer 2000;89:1555-60.

26. Gadducci A, Landoni F, Sartori E, et al. Analysis of treatment failures and survival of patients with fallopian tube carcinoma: A cooperation task force (ctf) study. Gynecol Oncol 2001;81:150-9.

27. Ferriss JS, Java JJ, Bookman MA, et al. Ascites predicts treatment benefit of bevacizumab in front-line therapy of advanced epithelial ovarian, fallopian tube and peritoneal cancers: An nrg oncology/gog study. Gynecol Oncol 2015;139:17-22.

28. Liu L, Xu X, Jia L, et al. Primary fallopian tube carcinoma-a retrospective analysis of 66 cases. Eur J Gynaecol Oncol 2015;36:161-7.

29. Ay C, Posch F, Kaider A, et al. Estimating risk of venous thromboembolism in patients with cancer in the presence of competing mortality. J Thromb Haemost 
2015;13:390-7.

30. Miller ME, Elashoff DA, Abemayor E, et al. Tonsillar squamous cell carcinoma: Are we making a difference? Otolaryngol Head Neck Surg 2011;145:236-41.

31. Rosen AC, Ausch C, Hafner E, et al. A 15-year overview of management and prognosis in primary fallopian tube carcinoma. Austrian cooperative study group for fallopian tube carcinoma. Eur J Cancer 1998;34:1725-9.

32. Jin C, Deng X, Li Y, et al. Lymph node ratio is an independent prognostic factor for rectal cancer after neoadjuvant therapy: A meta-analysis. J Evid Based Med 2018;11:169-175.

33. Persiani R, Cananzi FC, Biondi A, et al. Log odds of positive lymph nodes in colon cancer: A meaningful ratiobased lymph node classification system. World J Surg 2012;36:667-74.

34. Spolverato G, Ejaz A, Kim Y, et al. Prognostic performance of different lymph node staging systems after curative intent resection for gastric adenocarcinoma. Ann

Cite this article as: Li C, Li J, Huang Q, Feng X, Zhao F, $\mathrm{Xu}$ F, Han D, Lyu J. Developing and validating a novel nomogram used a competing-risks model for predicting the prognosis of primary fallopian tube carcinoma: a retrospective study based on the SEER database. Ann Transl Med 2021;9(5):378. doi: 10.21037/atm-20-5398
Surg 2015;262:991-8.

35. Wang J, Hassett JM, Dayton MT, et al. The prognostic superiority of log odds of positive lymph nodes in stage iii colon cancer. J Gastrointest Surg 2008;12:1790-6.

36. Hyder O, Marques H, Pulitano C, et al. A nomogram to predict long-term survival after resection for intrahepatic cholangiocarcinoma: An eastern and western experience. JAMA Surg 2014;149:432-8.

37. Cooperberg MR, Hilton JF, Carroll PR. The capra-s score: A straightforward tool for improved prediction of outcomes after radical prostatectomy. Cancer 2011;117:5039-46.

38. Lacy MQ, Hartmann LC, Keeney GL, et al. C-erb b-2 and $\mathrm{p} 53$ expression in fallopian tube carcinoma. Cancer 1995;75:2891-6.

39. Zheng W, Sung CJ, Cao P, et al. Early occurrence and prognostic significance of $\mathrm{p} 53$ alteration in primary carcinoma of the fallopian tube. Gynecol Oncol 1997;64:38-48. 